\title{
High polymorphism in MHC-DRB genes in golden snub-nosed monkeys reveals balancing selection in small, isolated populations
}

Pei Zhang ${ }^{1 \dagger}$, Kang Huang ${ }^{1 \dagger}$, Bingyi Zhang ${ }^{1}$, Derek W. Dunn ${ }^{1}$, Dan Chen ${ }^{2}$, Fan Li ${ }^{1}$, Xiaoguang Qi ${ }^{1}$, Songtao Guo ${ }^{1}$ and Baoguo $\mathrm{Li}^{1,3^{*}}$ (D)

\begin{abstract}
Background: Maintaining variation in immune genes, such as those of the major histocompatibility complex (MHC), is important for individuals in small, isolated populations to resist pathogens and parasites. The golden snub-nosed monkey (Rhinopithecus roxellana), an endangered primate endemic to China, has experienced a rapid reduction in numbers and severe population fragmentation over recent years. For this study, we measured the DRB diversity among 122 monkeys from three populations in the Qinling Mountains, and estimated the relative importance of different agents of selection in maintaining variation of DRB genes.

Results: We identified a total of $19 D R B$ sequences, in which five alleles were novel. We found high DRB variation in $R$. roxellana and three branches of evidence suggesting that balancing selection has contributed to maintaining MHC polymorphism over the long term in this species: i) different patterns of both genetic diversity and population differentiation were detected at MHC and neutral markers; ii) an excess of non-synonymous substitutions compared to synonymous substitutions at antigen binding sites, and maximum-likelihood-based random-site models, showed significant positive selection; and iii) phylogenetic analyses revealed a pattern of trans-species evolution for DRB genes.

Conclusions: High levels of DRB diversity in these $R$. roxellana populations may reflect strong selection pressure in this species. Patterns of genetic diversity and population differentiation, positive selection, as well as trans-species evolution, suggest that pathogen-mediated balancing selection has contributed to maintaining MHC polymorphism in $R$. roxellana over the long term. This study furthers our understanding of the role pathogen-mediated balancing selection has in maintaining variation in MHC genes in small and fragmented populations of free-ranging vertebrates.
\end{abstract}

Keywords: MHC, Rhinopithecus roxellana, Genetic diversity, Balancing selection

\footnotetext{
* Correspondence: baoguoli@nwu.edu.cn

${ }^{\dagger}$ Equal contributors

'Shaanxi Key Laboratory for Animal Conservation, College of Life Sciences,

Northwest University, Xi'an, China

${ }^{3}$ Xi'an Branch of Chinese Academy of Science, Xi'an, China

Full list of author information is available at the end of the article
} 


\section{Background}

The major histocompatibility complex $(M H C)$ gene family is one of the most polymorphic gene regions yet found in any vertebrate genome [1]. This multigene family plays a central role in the immune systems of many vertebrates by first recognizing foreign antigens and then binding and presenting them to $\mathrm{T}$ cells, thus triggering an appropriate immune response [2, 3]. Specifically, MHC class $I$ and II genes encode cell surface glycoproteins that bind intracellular (such as viruses) and extracellular foreign peptides (such as bacteria and parasites), respectively $[4,5]$. The class $I I$ gene series $D P, D Q$, and $D R$ encode heterodimeric proteins each with $\alpha$ and $\beta$ chains. The $D R \beta(D R B)$ genes, especially exon 2 , which encodes functionally important antigen binding sites (ABS), has been extensively studied in mammals [6-8]. Variation in residues within the ABS of different $M H C$ alleles defines the range of antigens that the immune system can identify and fight-off [9]. Thus, pathogenmediated balancing selection is a major agent shaping $M H C$ polymorphism as a consequence of an arms-race between pathogens and the host's immune system [9].

Three forms of balancing selection that may maintain variation in the $M H C$ have been recognized: heterozygote advantage, negative frequency dependent selection, and fluctuating selection $[1,10,11]$. All three forms of balancing selection may act simultaneously in maintaining $M H C$ variation.

Three methods are often used to detect balancing selection. First, natural selection can be estimated via calculating the selection parameter $\omega\left(d_{\mathrm{N}} / d_{\mathrm{S}}\right.$, the rate of non-synonymous substitutions/synonymous substitutions) and is the most common method used [11, 12]. According to the neutral selection theory, $\omega$ should not significantly deviate from one [13]. When $\omega$ is significantly greater than one this indicates positive selection, and in the case of $M H C$ genes, balancing selection due to host/pathogen coevolution [14]. Conversely, when $\omega$ is significantly less than one, this indicates negative/purifying selection [15]. Second, trans-species evolution is another common method used to identify historical balancing selection on $M H C$ genes, in which the same advantageous $M H C$ alleles are conserved across distinct evolutionary units in spite of differentiating evolutionary processes [16]. Third, different patterns of genetic diversity and population differentiation for genes under selection compared to neutral genes, can also indicate the presence of balancing selection [17].

In practice, the role of balancing selection in maintaining adaptive variation in the $M H C$ is still unclear, because various evolutionary factors can affect $M H C$ variation and may mask any effects of balancing selection [18]. More specifically, in small and/or fragmented populations, genetic drift is likely to reduce $M H C$ diversity $[19,20]$.
The golden snub-nosed monkey (Rhinopithecus roxellana) is an endangered primate endemic to China. Although once widespread in China, wild $R$. roxellana populations now only occur in fragmented populations in Sichuan, Gansu, Hubei and Shaanxi provinces. This study was conducted in the Qinling Mountains, Shaanxi province, the major east-west mountain range of China. These mountains provide a natural boundary between northern and southern China, and support much biodiversity. Within the Qinling Mountains, there are 39 known $R$. roxellana populations comprising a total of $\sim$ 4000 individuals [21]. The average population size is about 100 individuals. Each population concluded a breeding band, an all-male band and several solitary males [22]. Normally, males are able to migrate between neighboring populations $(<5 \mathrm{~km})$. While females often stay in their natal population, they can also migrate to neighboring populations via seasonal fission-fussion [22]. However, over the past few decades, suitable habitat for this species has decreased rapidly in both quality and quantity, and has become fragmented due to commercial logging, and the building of roads or other infrastructures [21]. The effects of fragmentation have also been exacerbated through human activities such as increased tourism, hunting, agricultural expansion, herb collecting, and firewood collection [21]. This has resulted in a reduction of the total $R$. roxellana population by more than $50 \%$ over the past 40 years, with $R$. roxellana being classified as endangered since 2008 by the IUCN [23].

Small and/or fragmented populations will experience reduced genetic variation due to inbreeding, restricted gene flow and genetic drift [24]. The degree of genetic variation is thought to facilitate the potential of small and/or fragmented populations to adapt to environmental change. Maintaining genetic variation in such populations is thus a critical component in appropriate conservation strategies for endangered species such as $R$. roxellana [25].

Previous studies of $R$. roxellana populations using neutral markers (such as mitochondrial D-loops and microsatellites) to assess genetic diversity have provided much information on phylogenetic relationships and demographic history [26, 27]. For example, microsatellite analysis revealed relatively high levels of both genetic diversity and population subdivision of Qinling Mountains $R$. roxellana [28]. Unfortunately, neutral markers cannot provide direct information associated with the ability and capacity of hosts resisting continuously evolving parasites and pathogens in the natural environment. To date, the adaptive nature of $M H C$ variation under pathogen-mediated coevolution in different Qinling Mountains $R$. roxellana populations is unknown.

In this study, we i) measure genetic variation of $M H C$ genes and microsatellite loci in three Qinling Mountains $R$. roxellana populations, ii) identify different agents of 
selection (including positive selection and trans-species evolution) and the roles they may play in maintaining variation in $M H C$ diversity, and iii) evaluate the potential for balancing selection for $M H C$ genes in $R$. roxellana populations. This study furthers our understanding of pathogen-mediated balancing selection at $M H C$ genes in small and/or fragmented populations of free-ranging vertebrates.

\section{Methods}

\section{Sampling and DNA extraction}

A total of 122 wild $R$. roxellana samples were collected from three populations in the Qinling Mountains, including 32 hair samples from Foping (FP), 36 fecal samples from Ningshan (NS), and 54 hair samples from Zhouzhi (ZZ) (Table 1). These populations were located in three officially protected nature reserves in three counties (Fig. 1). The FP and NS populations were located on the southern slope of the Qinling Mountains, whereas the ZZ population was located on the northern slope. The three study populations belong to three different reserves (Fig. 1). Long distances and habitat fragmentation prevent monkeys from migrating between these three populations [28].

Hair samples were collected using a pole with glue on its end. Each sample was stored individually at room temperature in a labeled envelope in a dryer containing desiccant granules. Fresh fecal samples were stored in DMSO salt solution (DETs: 20\% DMSO, $0.25 \mathrm{M}$

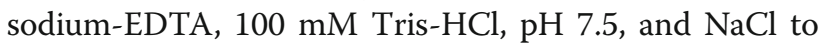
saturation) at $-20{ }^{\circ} \mathrm{C}$. Both individual identification and sample collections complied with the animal welfare laws and constitutions of China. DNA was extracted from hair samples according to a Chelex protocol (Chelex 100, Bio-Rad) [29]. Fecal DNA was extracted using QIAamp DNA Stool Mini Kits (Qiagen, Germany). Individuals were identified via microsatellite profiles with 19 loci to exclude repeated samples from a same individual [28]. During the extraction and subsequent polymerase chain reaction (PCR), laboratory benches were washed with $75 \%$ ethanol. All facilities and disposable plasticware used were exposed to UV light for $30 \mathrm{~min}$ prior to

Table 1 The location, population size, number of samples and sampling time of each study population

\begin{tabular}{llllll}
\hline Population & Reserve & Location & Size & Samples $^{\text {a }}$ & Sampling time \\
\hline $\begin{array}{l}\text { Foping } \\
\text { (FP) }\end{array}$ & FNNR & $\begin{array}{l}33.68^{\circ} \mathrm{N}, \\
107.99^{\circ} \mathrm{E}\end{array}$ & & $32 / 0$ & November 2014 \\
$\begin{array}{l}\text { Ningshan } \\
\text { (NS) }\end{array}$ & NNR & $\begin{array}{l}33.66^{\circ} \mathrm{N}, \\
108.35^{\circ} \mathrm{E}\end{array}$ & 100 & $0 / 36$ & January 2015 \\
$\begin{array}{lllll}\text { Zhouzhi } \\
\text { (ZZ) }\end{array}$ & ZNNR & $\begin{array}{l}33.79^{\circ} \mathrm{N}, \\
108.26^{\circ} \mathrm{E}\end{array}$ & & & \\
& & & & & \\
\end{tabular}

${ }^{\mathrm{a} N u m b e r s ~ i n ~ t h e ~ f r o n t ~ o f ~ a n d ~ b e h i n d ~ t h e ~ s l a s h ~ d e n o t e d ~ t h e ~ h a i r ~ a n d ~ f e c e s ~}$ samples collected, respectively. Size, estimated population size; FNNR, Foping National Nature Reserve; NNR, Ningshan Nature Reserve; ZNNR, Zhouzhi National Nature Reserve

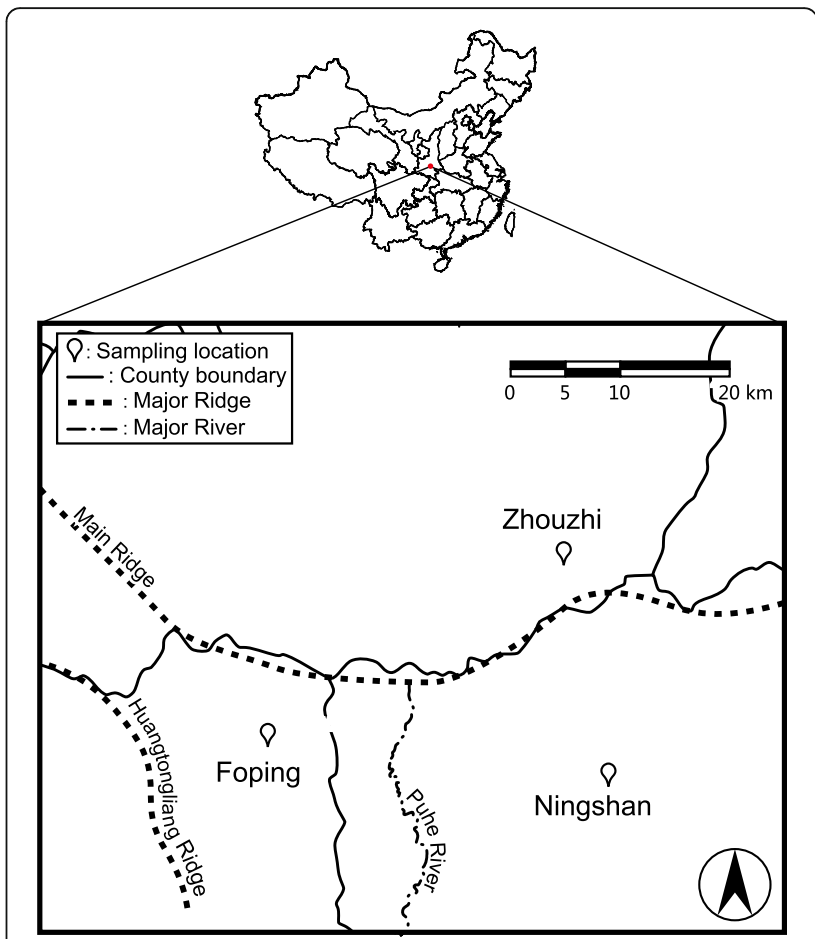

Fig. 1 Distribution of the three $R$. roxellana populations used for this study. The range of coordinates were $107.8^{\circ}-108.5^{\circ} \mathrm{E}, 33.5^{\circ}-34.0^{\circ} \mathrm{N}$, UTM projection

treatment, preventing contamination by human DNA. For the same purpose, negative controls were used for each PCR reaction.

\section{Molecular techniques}

Overall genetic diversity was assessed based on 19 microsatellites. All individuals were genotyped at these microsatellites following the previously established methods [28], using the same primers as used in Huang et al. [28]. Adaptive variation was studied in the highly polymorphic $D R B$ exon 2 fragments. To amplify the exon 2 of the $D R B$ genes in $R$. roxellana, a primer pair (F: 5'-TTCTCAGGAGGCCGCCCGTGTGA-3'; R: 5'ACCTCGCCGCTGCACTGTGAAGCTC-3') was used [30]. The length of $D R B$ exon 2 is 270 base pairs (bp). The primer pair amplified a 270 bp product, which contains 247 bp of exon 2 and 23 bp of intron 1. PCR was performed in $50 \mu \mathrm{L}$ of reaction mix containing $10 \mathrm{mM}$ Tris- $\mathrm{HCl}$ (pH 8.4), $50 \mathrm{mM} \mathrm{KCl}, 2.5 \mathrm{mM} \mathrm{MgCl} 2,0.4 \mu \mathrm{M}$ of forward and reverse primers, $0.2 \mathrm{mM}$ of each dNTP, 1 unit of ExTaq DNA polymerase (Takara, Dalian), and 10-100 ng of genomic DNA. Amplification was carried out in a Veriti ${ }^{\text {tw }}$ 96-Well Fast Thermal Cycler (Applied Biosystems, Singapore) under the following conditions: initial denaturation at $94{ }^{\circ} \mathrm{C}$ for $5 \mathrm{~min}$, followed by $35 \mathrm{cy}$ cles of denaturation at $94{ }^{\circ} \mathrm{C}$ for $30 \mathrm{~s}$, annealing at $60{ }^{\circ} \mathrm{C}$ 
for $30 \mathrm{~s}$ and extension at $72{ }^{\circ} \mathrm{C}$ for $30 \mathrm{~s}$, finishing with a final extension at $72{ }^{\circ} \mathrm{C}$ for $10 \mathrm{~min}$.

Amplification products were purified using an AxyPrep ${ }^{\mathrm{Tx}}$ DNA Gel Extraction Kit (AXYGEN Biosciences) according to the manufacturer's protocol. Purified PCR product was then ligated into a pMD 18-T Vector (Tarkara, Dalian) and transformed into a DH5 $\alpha$ competent cell (Tarkara, Dalian). Twenty positive clones containing inserts from each individual were sequenced in both directions using an ABIPrism $^{\text {Tw }} 3100$ Genetic Analyzer (Applied Biosystems Inc.).

\section{Data analysis}

\section{Identification of MHC alleles}

All sequences were aligned by CLUSTALX V2.0 [31]. To prevent interference of the PCR amplification artifacts, each new sequence was considered to be an allele if it had been detected in at least two different individuals or in three different PCRs of the same individual. Then, each allele was aligned with Rhro-DRB*01-37 (GenBank accession numbers: JQ863322-JQ863358) [30] and verified with the whole genomic data of $R$. roxellana [32] using the BLAST (Basic Local Alignment Search Tool; https://blast.ncbi.nlm.nih.gov/Blast.cgi) of the NCBI.

Because our sampling was limited to a narrow time window (Table 1) only samples from adult individuals were obtained. Our data therefore did not include any known parent-offspring relationships (parent-offspring duos, or mother-father-offspring trios) to validate the observed individual $D R B$ genotypes. We used MHC-TYPER V1.0 (unpublished, https://github.com/huangkang1987/ mhc-typer), a new method for assigning alleles to different loci based on the calculation of likelihood of loci, to assign each allele to a specific locus.

MHC-TYPER V1.0 uses a simulated annealing algorithm to find the optimal allele configuration, which is defined as a partition of the alleles into several loci [33]. The searching procedure begins from a trivial initial solution (i.e. allele configuration), then randomly mutates to a new solution. The new solution will be accepted according to a ratio that depends on current temperature and the difference in the evaluation value (AIC or BIC) between the new and the current solutions. Both parameters are functions of the likelihood of the genotypes calculated from the allele configuration. If the current temperature is high, the same non-optimal solution will be accepted at a high ratio. Therefore, the searching algorithm randomly 'walks' across configurations with high temperatures and becomes increasingly 'greedy' (accepts worse configurations at a low probability) as the temperature decreases. The simulated annealing algorithm simulates the annealing of a metal, and begins from a relatively high temperature, and decreases by being repeatedly multiplied by an annealing coefficient of less than one (e.g. 0.99). The annealing cycle is repeated several times to ensure that the optimal solution is found.

\section{Genetic variation at MHC and microsatellites}

BEV81148xDRB sequences were translated into amino acid sequences using MEGA V7 [34]. Variable sites, parsimony-informative sites, and overall mean genetic distances of nucleotide sequences were derived in MEGA V7.0 [34]. Deviation from the Hardy-Weinberg equilibrium (HWE) was tested with 100,000 steps of Markov chain using GENEPOP V4.3 [35] for each population. Allelic Richness $\left(A_{\mathrm{R}}\right)$ based on minimal sample size and inbreeding coefficient $\left(F_{\mathrm{IS}}\right)$ per locus were both calculated using FSTAT V2.9.3 [36]. Expected heterozygosity $\left(H_{\mathrm{E}}\right)$, observed heterozygosity $\left(H_{\mathrm{O}}\right)$, polymorphism information content (PIC) and the frequency of null alleles $\left(P_{\text {null }}\right)$ were calculated using CERVUS V3.0 [37]. The effective number of alleles $\left(A_{\mathrm{E}}\right)$ per locus and $F$-statistics $\left(F_{\mathrm{ST}}\right)$ were both computed using GENALEX V6.5 [38]. In addition, Tajima's test was conducted using DNASP V5.10.01 [39]. A positive Tajima's $D$ value means a heterozygous advantage or population reduction, while a negative value represents selection on a specific allele or population expansion [40].

To test whether $M H C$ loci were behaving differently from neutral loci, allelic richness $\left(A_{\mathrm{R}}\right)$, inbreeding coefficient $\left(F_{\mathrm{IS}}\right)$, expected heterozygosity $\left(H_{\mathrm{E}}\right)$, observed heterozygosity $\left(H_{\mathrm{O}}\right)$, polymorphism information content (PIC) and population differentiation $\left(F_{\mathrm{ST}}\right)$ were estimated for both $M H C$ and microsatellite loci. We used Mann-Whitney $U$ test to compare the $H_{\mathrm{E}}$ and PIC between these two types of loci. Data were analyzed using SPSS V22.0 (IBM). All $P$-values are two tailed, and the level significance was 0.05 .

\section{Phylogenetic analysis}

Phylogenetic relationships among Rhro-DRB alleles (including data from previous research [30]) (GenBank accession numbers: JQ217116-JQ217131) were reconstructed using Ovar-DRB1*0101 (DRB1*0101 allele of Ovis aries, GenBank accession number: Y07898) as outgroups. Orthologous sequences from Macaca fascicularis (Mafa-DRB), Macaca mulatta (Mamu-DRB), Mandrillus sphinx (Masp-DRB),

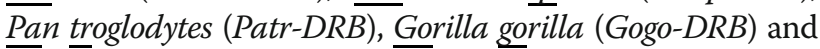
Homo sapiens (HLA-DRB) (GenBank accession numbers: KF880641, KF880647, M96121.1, M96122.1, DQ103723, DQ103724, DQ103725, DQ103732.1, AM086033, AM086040, AF031254, AF031271.1, FJ442950.1, DQ837166.1, EU934775.1, FN424202.1, HE800526.1, KR632831.1， HM580015.1， HM594301， FR717382, EF208835 and JQ579493.1) were included in the analysis. Best-fit models for nucleotide substitution (assuming a gamma distribution) were determined using the Akaike Information Criterion (AIC) in JMODELTEST V2.1.3 [41]. 
Phylogenetic relationships were then estimated according to the Bayesian approach using MRBAYES V3.0 [42], the maximum likelihood (ML) method using PHYML V3.0 [43] and the maximum parsimony (MP) method using MEGA V7 [34], respectively. The reliability of each tree topology structure was carried out via 1000 bootstrap replications.

\section{Selection pressure analysis}

We calculated $\omega$ at all amino acid sites, ABS, and nonABS in the exon 2 region in MEGA V7 [34] using the NeiGojobori method with the Jukes-Cantor correction [44] and 1000 bootstrap replicates to obtain standard errors. The putative ABS and non-ABS were both derived according to the structure of human $D R B$ genes [45]. The statistical significance of the difference between $d_{\mathrm{N}}$ and $d_{\mathrm{S}}$ was tested using a $Z$-test implemented in MEGA V7 [34]. Evidence for natural selection was also obtained using the CODEML program in PAML V4.7 [46]. The heterogeneity of $\omega$ among codons was examined based on the maximum likelihood method. Six models (M0, M1a, M2a, M3, M7 and M8) allowing different selection intensity among sites were compared using likelihoodratio tests in PAML V4.7 [12, 46]. Posterior probabilities for site classes were calculated by the Bayes empirical Bayes (BEB) method in models M2a and M8.

\section{Results}

\section{$D R B$ allele assignment}

We examined the variation of $D R B$ genes of $122 R$. roxellana monkeys from three populations (FP, NS and ZZ). Twenty clones were sequenced from both strands for each individual. Nineteen different $D R B$ sequences were obtained with $270 \mathrm{bp}$, including $247 \mathrm{bp}$ exon 2 and partial intron 1.

After performing alignments with Rhro-DRB*01-37 (GenBank accession numbers: JQ863322-JQ863358) [30], fourteen sequences were matched with Rhro$D R B^{*} 02,03,04,05,06,07,08,09,14,16,17,18,23$ and 26 , respectively. Five novel $D R B$ sequences were identified and labeled as Rhro-DRB*38, 39, 40, 41 and 42 (GenBank accession numbers: MF434639-MF434643) according to the nomenclature reported by Klein et al. [47]. Each of the new alleles identified in this study was present in at least three different PCRs, without stop codons, frame-shift mutations, or insertions/deletions. Rhro-DRB*23, first detected by Luo and Pan [30], lost three bases, which also resulted in one amino acid deletion. Two to four alleles were detected in each individual, indicating at least two loci were amplified, which was consistent with previous research [30]. According to the results of MHC-TYPER V1.0, 11 alleles were assigned to Rhro-DRB1 and 8 alleles were assigned to Rhro-DRB2 (Rhro-DRB1: 02, 03, 06, 09, 14, 17, 23, 38, 39, 41 and 42; Rhro-DRB2: 04, 05, 07, 08, 16, 18, 26 and 40).

\section{Genetic variation at $D R B$ and microsatellites}

DNA extracts were amplified at 19 microsatellite loci and two $D R B$ genes. The characteristics of these loci are presented in Tables 2, 3 and 4. The number of alleles per microsatellite locus ranged from 3 to 6 , with an average of 4.6. The number of alleles within the two $D R B$ loci varied among the three study populations, ranging from 10 in FP to 14 in ZZ (Table 3). Some of these alleles were abundant (more than $30 \%$ ) in all three populations (e.g., DRB 03 and $D R B * 04$ ), whilst others were detected at low frequencies (less than 10\%) and/or only in one population (e.g., $D R B^{*} 18,23,26,38,39,40,41$ and 42).

The $H_{\mathrm{O}}$ are generally lower than $H_{\mathrm{E}}$ in these three populations at both $D R B$ loci, with the exception of the $D R B 2$ locus in the FP population (Table 4). For microsatellite loci, the $H_{\mathrm{O}}$ are all higher than $H_{\mathrm{E}}$ in three populations (Table 2). We only observed significant deviations from HWE (Bonferroni correction, $P=0.008$, Table 4 ) and an excess of homozygotes at the $D R B 2$ locus in the $\mathrm{ZZ}$ population $\left(H_{\mathrm{O}}=\right.$ $\left.0.415 ; H_{\mathrm{E}}=0.640\right)$, in which a high frequency of null alleles was detected $\left(P_{\text {null }}=0.202\right)$. The levels of $H_{\mathrm{E}}$ were all above 0.5 among the three populations at two loci (DRB1: 0.581$0.749 ;$ DRB2: $0.524-0.640)$ and 19 microsatellite loci (0.512-0.535), indicating a high level of genetic diversity at both types of markers in all three populations. The values of PIC were generally over 0.5 for MHC genes, with the exception of the DRB2 gene in the FP population (0.479), suggesting that other combinations had high polymorphism. For microsatellites, the values of PIC were all less than 0.5 (0.451-0.466), indicating that microsatellites had moderate polymorphism. Allelic richness $\left(A_{\mathrm{R}}\right)$ also varied at two types of loci, with $D R B 1$ ranging from 5.994 to $6.993, D R B 2$ ranging from 4.000 to 5.993 , with microsatellites ranging from 3.340 to 3.606 (Tables 2 and 4).

The genetic diversity of $M H C$ loci (average $H_{\mathrm{E}}=0.626$, $\mathrm{PIC}=0.583$ ) is higher than that of microsatellites (average $H_{\mathrm{E}}=0.527, \mathrm{PIC}=0.459$ ). Mann-Whitney $U$ tests showed that the difference in $H_{\mathrm{E}}$ between two types of loci are not significantly different $\left(n_{1}=60, n_{2}=6, U=\right.$ 115.5, $P=0.194)$, whereas the PIC of $M H C$ loci are significantly higher than that of microsatellites $(U=87.0$, $P=0.049$ ).

Table 2 Population genetic parameters for three populations estimated from microsatellite data

\begin{tabular}{llllllll}
\hline Population & No. of Loci & $k$ & $A_{R}$ & PIC & $H_{\mathrm{O}}$ & $H_{\mathrm{E}}$ & $F_{\mathrm{IS}}$ \\
\hline FP & 19 & 3.58 & 3.340 & 0.466 & 0.496 & 0.535 & 0.026 \\
NS & 19 & 3.74 & 3.606 & 0.451 & 0.506 & 0.512 & -0.075 \\
ZZ & 19 & 3.68 & 3.457 & 0.461 & 0.514 & 0.534 & 0.038 \\
All & 19 & 4.63 & 3.839 & 0.520 & 0.531 & 0.581 & - \\
\hline
\end{tabular}

$k$ mean number of alleles for all loci, $A_{R}$ allelic richness, PIC polymorphic information content, $H_{\mathrm{O}}$ and $H_{\mathrm{E}}$, observed and expected heterozygosity, respectively; $F_{\mathrm{IS}}$, inbreeding coefficient 
Table 3 Allele frequencies of $M H C$ in three $R$. roxellana populations in the Qinling Mountains

\begin{tabular}{|c|c|c|c|c|c|}
\hline Locus & Allele & $\begin{array}{l}\mathrm{FP}(6 / 4)^{\mathrm{a}} \\
N=32\end{array}$ & $\begin{array}{l}\text { NS }(7 / 6)^{a} \\
N=36\end{array}$ & $\begin{array}{l}Z Z(8 / 6)^{a} \\
N=54\end{array}$ & $\begin{array}{l}\text { All }(11 / 8)^{a} \\
N=122\end{array}$ \\
\hline \multirow[t]{11}{*}{ DRB1 } & $D R B^{*} 02$ & - & 0.042 & 0.074 & 0.045 \\
\hline & $D R B^{*} 03$ & 0.625 & 0.583 & 0.370 & 0.500 \\
\hline & $D R B^{*} 06$ & 0.031 & 0.083 & - & 0.033 \\
\hline & $D R B^{*} 09$ & 0.031 & 0.083 & 0.213 & 0.127 \\
\hline & $D R B^{*} 14$ & 0.125 & 0.083 & 0.259 & 0.172 \\
\hline & $D R B^{*} 17$ & 0.125 & 0.083 & 0.046 & 0.078 \\
\hline & $D R B^{*} 23$ & - & 0.042 & - & 0.012 \\
\hline & $D R B^{*} 38$ & - & - & 0.019 & 0.008 \\
\hline & $D R B^{*} 39$ & - & - & 0.009 & 0.004 \\
\hline & $D R B^{*} 41$ & 0.063 & - & - & 0.016 \\
\hline & $D R B^{*} 42$ & - & - & 0.009 & 0.004 \\
\hline \multirow[t]{8}{*}{ DRB2 } & $D R B^{*} 04$ & 0.667 & 0.583 & 0.519 & 0.576 \\
\hline & $D R B^{*} 05$ & 0.067 & 0.125 & 0.075 & 0.088 \\
\hline & $D R B^{*} 07$ & - & - & 0.038 & 0.017 \\
\hline & $D R B^{*} 08$ & 0.133 & 0.042 & 0.292 & 0.177 \\
\hline & $D R B^{*} 16$ & 0.133 & 0.125 & 0.066 & 0.101 \\
\hline & $D R B^{*} 18$ & - & 0.083 & - & 0.025 \\
\hline & $D R B^{*} 26$ & - & 0.042 & - & 0.013 \\
\hline & $D R B^{*} 40$ & - & - & 0.009 & 0.004 \\
\hline
\end{tabular}

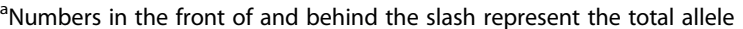
number of $D R B 1$ and $D R B 2$, respectively. $\mathrm{N}$ represents sample size in certain population

We identified 67 (27.1\%) variable nucleotide positions in a $247 \mathrm{bp}$ sequence. These sequences showed wideranging levels of divergence with an average of 24.7 (10\%) nucleotide differences (minimum: 3 substitutions, maximum: 37 substitutions) among sequences. We detected $33(40.1 \%)$ variable positions in the 81 amino acid sequence. The number of pairwise amino acid differences between sequences ranged from 2 to 22 with an average of 15.3 (18.9\%). For locus-specific variation, we found $60(24.7 \%)$ variable nucleotides for $D R B 1$ and 44 (18.1\%) for $D R B 2$, corresponding to $30(37.0 \%)$ amino acid residue changes in DRB1 and 23 (28.4\%) changes in $D R B 2$ (Table 4; Additional file 1: Figure S1). The proportion of variable amino acids at the ABS for both loci exceeded 50\% (16/18 in DRB1 and 12/18 in DRB2), with the overall mean distance $0.104 \pm 0.015$ at the $D R B 1$ locus and $0.082 \pm 0.014$ at $D R B 2$. Thus, both loci exhibit high levels of sequence divergence.

Among populations, the FP population had the lowest polymorphism at two $D R B$ loci for most parameters (Table 4). The number of alleles (DRB1: 6; DRB2: 4), the variable nucleotide sites (DRB1: 48; DRB2: 37), the PIC (DRB1: 0.542; DRB2: 0.479) and $H_{\mathrm{E}}$ (DRB1: 0.581; DRB2: $0.524)$ in the FP population were all lower than in the other two populations (Table 4). However, the observed heterozygosity $\left(H_{\mathrm{O}}=0.415\right)$ at $\mathrm{DRB} 2$ locus in the $\mathrm{ZZ}$ population is lower than the other combinations (Table 4). We also found that the $M H C$ allele frequencies of different populations were less differentiated than microsatellite allele frequencies (Table 5). $F_{S T}$ values of adaptive $M H C$ genes and neutral microsatellites among the three study populations are shown in Table 5.

\section{Positive selection}

The selection parameter $\omega\left(d_{N} / d_{S}\right.$, the rate of nonsynonymous substitutions/synonymous substitutions) was calculated for the ABS, non-ABS and all amino acid positions (Table 6). For the ABS sites across all alleles, $\omega$ was significantly greater than one $(\omega=6.807, P=0.000)$, indicating that there was a strong positive selection at these sites in the Rhro-DRB sequences. For the non-ABS sites, $\omega$ was less than one $(\omega=0.505, P=0.013)$, suggesting negative/purifying selection at the non-ABS sites.

Amino acid residues under significant positive selection were also found with PAML V4.7 (Table 7) using the maximum likelihood method. Various codon evolutionary models were compared using CODEML program in PAML V4.7 [46]. With regard to the Akaike Information Criterion (AIC) values, models integrating positive selection (M2a, M3, and M8) matched MHC better than the other models (Tables 7 and 8). Under models M2a and M8, two sites (11F and 13S) were exposed to significant selection. Under model M3, 32 sites were identified, in which 27 sites (9E, 10Q, 11F, 13S, 26Y, 28Q, 30Y, 31F, 32Y, 37Y, $38 \mathrm{~V}, 47 \mathrm{~F}, 49 \mathrm{~A}, 56 \mathrm{P}, 57 \mathrm{~V}, 60 \mathrm{~N}, 61 \mathrm{~F}, 64 \mathrm{Q}, 67 \mathrm{~F}, 70 \mathrm{Q}, 71 \mathrm{R}$, $72 \mathrm{R}, 74 \mathrm{Q}, 77 \mathrm{~N}, 78 \mathrm{Y}, 84 \mathrm{G}$ and $86 \mathrm{~V}$ ) including both $11 \mathrm{~F}$ and 13S, showed significant selection pressure (Table 7). Moreover, most of these sites were associated with a peptide and/or a T-cell receptor (TCR) (Additional file 1: Figure S1). Collectively, these results indicate that in $R$. roxellana, most of the positive selection we identified occurred at functionally important sites.

The values for Tajima's $D$ statistics for the two $D R B$ loci of all three populations were all positive but did not differ significantly from the equilibrium neutral expectation (Table 4).

\section{Trans-species evolution}

In order to investigate the evolutionary relationships of $D R B$ sequences among $R$. roxellana and other primates, Bayesian, ML and MP phylogenetic trees were constructed (Fig. 2; Additional file 2: Figure S2). Ovar$D R B 1 * 0101$ was selected as the outgroup, and only bootstrap values/posterior probabilities greater than 50\% were included. This showed that consistent with transspecies evolution, the allelic relationships were inconsistent with the species relationships, and alleles from different species intermixed with each other. No clear clade for Rhro-DRB was identified in the phylogenetic tree. 


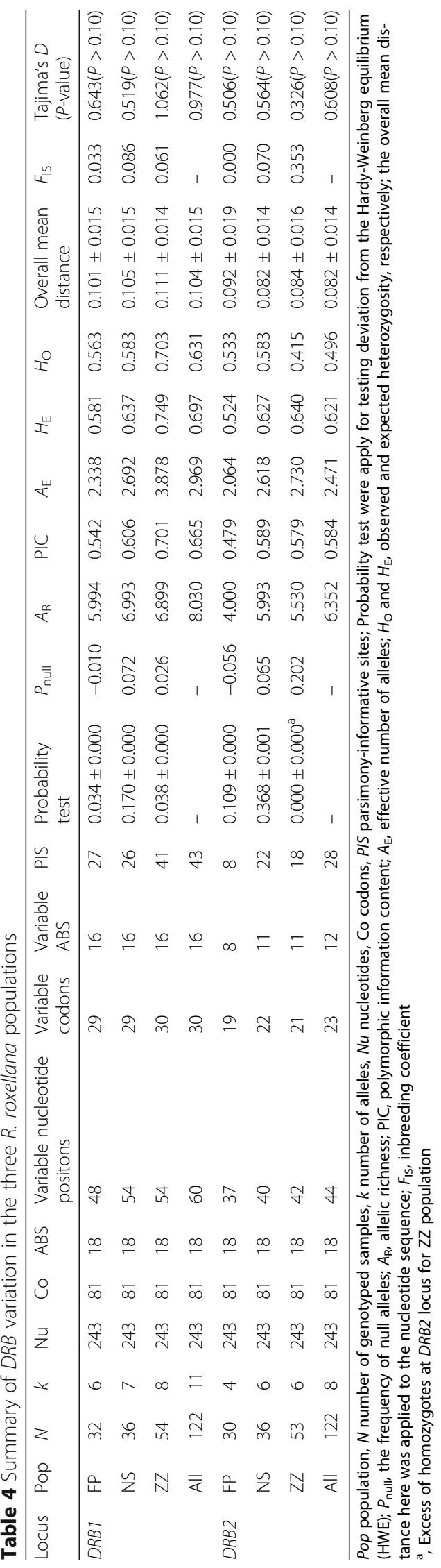


Table 5 Summary of population differential $\left(F_{\mathrm{ST}}\right)$ in the three $R$. roxellana populations

\begin{tabular}{llll}
\hline Locus & Population & FP & NS \\
\hline MHC & NS & 0.002 & \\
& ZZ & 0.051 & 0.053 \\
Microsatellite & NS & 0.108 & \\
& ZZ & 0.132 & 0.108 \\
\hline
\end{tabular}

\section{Discussion}

\section{Patterns of the Rhro-DRB diversity}

We measured the genetic variation of the Rhro-DRB genes of 122 individuals from three $R$. roxellana populations in the Qinling Mountains, and found 19 different $D R B$ alleles, of which five alleles were novel. Rhro$D R B * 23$, which lost three bases and resulted in one amino acid deletion, is considered a functional allele [30]. After performing BLAST on the NCBI website (https://blast.ncbi.nlm.nih.gov/Blast.cgi), we found several homologous sequences in other primate species which have lost the same three bases as has Rhro$D R B * 23$. Many of these sequences were expressional mRNA sequences, such as Mafa-DRB (GenBank accession numbers: HM580023, JQ579479, LT746055 and LT707677); Mamu-DRB (GenBank accession number: KF880635.1); Mane-DRB (Macaca nemestrina, GenBank accession number: JQ693980); Paur-DRB (Papio ursinus, GenBank accession number: DQ339731) and Caja-DRB (Callithrix jacchus, GenBank accession numbers: LN906590, LN906591 and LT908516). We thus conclude that $R$ hro-DRB*23 is functional and was present in the common ancestor of all species in which it is currently present.

Two to four alleles were detected within each individual, indicating at least two $D R B$ loci were sequenced in this study. In addition, a high similarity of alleles between two loci were found, suggesting that gene duplication plays a role in increasing copy numbers of $D R B$ genes. Gene duplication among $M H C$ genes has also been observed in other mammals [48-50]. Three of 19 alleles (16\%) were found only once among our samples, together with the detection of many novel alleles (five in 19). This suggests that there may be rapidly evolving loci within this species consistent with MHC class II genes in mammals that are found to have high duplication rates [51]. Given the high frequency (more than 30\%) of the

Table 6 Rate of non-synonymous substitutions $\left(d_{N}\right)$ and synonymous substitutions $\left(d_{s}\right)$

\begin{tabular}{lllll}
\hline Sites & $d_{N}$ & $d_{S}$ & $\omega$ & $P$ \\
\hline ABS & $0.388 \pm 0.071$ & $0.057 \pm 0.038$ & 6.807 & 0.000 \\
Non-ABS & $0.053 \pm 0.013$ & $0.105 \pm 0.034$ & 0.505 & 0.013 \\
All & $0.111 \pm 0.019$ & $0.094 \pm 0.026$ & 1.181 & 0.597 \\
\hline
\end{tabular}

alleles $D R B^{*} 03$ and 04 among all three $R$. roxellana populations used for this study, it is possible that these alleles may be subject to particular selective pressures that allow the sequences to persist longer than expected under neutrality [52].

The diversity of $M H C$ class II genes of several primate species has been investigated over the past two decades, especially those in the rhesus macaque (Macaca mulatta) $[53,54]$. This is an important model species in preclinical transplantation research and for the study of chronic and infectious diseases. Much of those research focused on the Mamu-DRB haplotype, and compared with humans, revealed high levels of polymorphism at the Mamu-DRB region configurations [53]. More recently, extensive research on non-model primate species have evaluated the adaptive nature of this genetic diversity. For example, 16 different $D R B$ sequences were detected in 30 chacma baboons (Papio ursinus) [55]. These exhibited wide-ranging divergence based on 92 (36.5\%) variable nucleotides in a $252 \mathrm{bp}$ sequence, and 40 (47.6\%) variable sites in a 84 amino acid sequence [55]. The mouse lemur (Microcebus murinus) also shows high levels of sequence divergence. In this species, 12 different $D R B$ alleles were found in 145 individuals, with 58 (33.9\%) variable positions in the $171 \mathrm{bp}$ sequence and 29 (50.9\%) variable positions out of 57 amino acids [56]. Schwensow et al. [57] also found much genetic variability in fat-tailed dwarf lemurs (Cheirogaleus medius), with 50 different $D R B$ alleles differing at one to 42 positions from each other, and $33(57.9 \%)$ out of 57 amino acid positions being variable. In this study, we found that the $D R B$ sequence divergence in the three study $R$. roxellana populations is relatively low compared with $M a$. mulatta, P. ursinus, Mi. murinus and C. medius. We identified $67(27.1 \%)$ variable nucleotide positions with an average of 24.7 (10\%) differences among sequences corresponding to $33(40.1 \%)$ variable amino acid positions with an average of 15.3 (18.9\%) differences among sequences were detected. The proportion of variable amino acids at the ABS for both loci is more than $50 \%$ (16/18 in DRB1 and $12 / 18$ in DRB2), meaning that most residual changes occurred in functionally important regions. This is similar to other $M H C$ loci not only in other primates species $[57,58]$, but also in other vertebrate species, such as the mummichog (fish) (Fundulus heteroclitus: [59]), the red-eyed tree frog (Agalychnis callidryas: [60]), the lesser kestrel (Falco naumanni: [61]) and the giant panda (Ailuropoda melanoleuca: [62]).

The PIC and $H_{\mathrm{E}}$ of two $D R B$ loci both exceed 0.5 (DRB1: $\mathrm{PIC}=0.665, H_{\mathrm{E}}=0.697 ; \mathrm{DRB2}: \mathrm{PIC}=0.584, H_{\mathrm{E}}=$ 0.621 ), indicating a high genetic diversity for $D R B$ genes, which is congruent with results obtained for two other $M H C$ genes $\left(D Q A 1\right.$ : $\mathrm{PIC}=0.662, H_{\mathrm{E}}=0.715$; and $D Q B 1$ : $\left.\mathrm{PIC}=0.658, H_{\mathrm{E}}=0.713\right) \quad$ [63]. In fact, our observed 
Table 7 Results of maximum likelihood models of the Rhro-DRB sequences

\begin{tabular}{|c|c|c|c|c|}
\hline Model & k & 1 & Parameters estimates & Positively selected sites \\
\hline MO (one ratio) & 1 & - & $\omega=0.559$ & None \\
\hline $\begin{array}{l}\text { M1a (nearly } \\
\text { neutral) }\end{array}$ & 1 & - & $p_{0}=0.603\left(p_{1}=0.397\right)$ & Not allowed \\
\hline $\begin{array}{l}\text { M2a (positive } \\
\text { selection) }\end{array}$ & 3 & - & $\begin{array}{l}p_{0}=0.576, p_{1}=0.400\left(p_{2}=0.025\right) \\
\omega_{2}=12.820\end{array}$ & $11 F, 13 S$ \\
\hline M3 (discrete) & 5 & $-\overline{1651.07}$ & $\begin{array}{l}p_{0}=0.602, p_{1}=0.374\left(p_{2}=0.025\right) \\
\omega_{0}=0.035, \omega_{1}=1.496, \omega_{2}=16.973\end{array}$ & 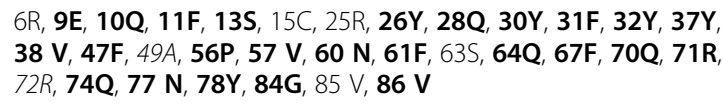 \\
\hline M7 (beta) & 2 & - & $p=0.079, q=0.125$ & Not allowed \\
\hline $\begin{array}{l}\text { M8 (beta } \\
\text { and omega) }\end{array}$ & 4 & -1651.44 & $p_{0}=0.975\left(p_{1}=0.025\right), p=0.019, q=0.035, \omega=11.880$ & $11 \mathrm{~F}, 13 \mathrm{~S}$ \\
\hline
\end{tabular}

$K$ represents the number of parameters in the $\omega$ distribution, $\omega$ is the parameter of selection, $p_{\mathrm{n}}$ is the proportion of sites falling into the $\omega_{\mathrm{n}}$ sites class. For models M7 and M8, $p$ and $q$ are the shape parameters of the $\beta$ function. Sites inferred under selection at the $99 \%$ level are listed in bold, and those inferred at the $95 \%$ level are shown in italics

polymorphism is likely to underestimate the variation across the entire exon 2 , given that our $D R B$ sequences covered only $247 \mathrm{bp}$ in the exon.

Among populations, the FP population had the lowest polymorphism for $D R B$ genes in most parameters except $H_{\mathrm{O}}$ (Table 4). The lowest $H_{\mathrm{O}}$ was in the $\mathrm{ZZ}$ population. The observed heterozygosity can be influenced by many factors, including inbreeding, selection, random effect, and null alleles. Inbreeding can result from non-random mating, including mating between closely related individuals, and pervasive inbreeding (due to genetic drift in a small or subdivided population) [64]. However, we found inbreeding coefficients at 19 microsatellites ranged from -0.075 to 0.038 , indicating there is little or no effect of inbreeding in each of the three study populations [65]. The observed degree of heterozygosity may also be affected by selection, which relies on fitness variation among individuals of different genotypes through both differential survival and differential reproduction (e.g. though non-random mating) [66, 67]. The low overall inbreeding coefficients in each population estimated from microsatellites suggest that non-random mating has little effect. Selection is thus unlikely to be the sole cause of the observed heterozygote deficiencies. Any random effects fail to explain the excess of homozygotes - our testing the hypothesis that $H_{\mathrm{O}}$ is equal to $H_{\mathrm{E}}$ and any difference is due to random error being rejected $(P=0.0007)$. We suggest that the most parsimonoious

Table 8 Likelihood-ratio test of codon evolution for exon 2 sequences at Rhro-DRB loci

\begin{tabular}{llll}
\hline Models compared & $\mathrm{d} f$ & Test statistic & Significance $(P)$ \\
\hline M2a vs. M1a & 2 & 74.6 & $<0.001$ \\
M3 vs. M0 & 4 & 358.04 & $<0.001$ \\
M8 vs. M7 & 2 & 70.8 & $<0.001$ \\
\hline
\end{tabular}

explanation for the excess of homozygotes in the $\mathrm{ZZ}$ population is the presence of null alleles. These are alleles that cannot be amplified, usually due to the mutations at the primers binding sites [68]. The frequency of null alleles at the DRB2 locus in the $\mathrm{ZZ}$ population is the highest out of the three study populations $\left(P_{\text {null }}=\right.$ 0.202), which increases the levels of observed homozygosity. Hence, the inbreeding coefficient in the $\mathrm{ZZ} \mathrm{popu-}$ lation is over-estimated, and the genotypic frequencies deviate significantly to those expected from the HWE at the DRB2 locus (Table 4).

\section{Evidence for balancing selection}

Key aspects of a species' adaptations to challenging environments are likely to have been pathogen-mediated [69]. Immunity-related $M H C$ is an ideal model gene family for studying host-pathogen coevolution [9]. Many wild populations have suffered from a reduction in $M H C$ diversity after past population decline [19, 70, 71]. However, in this study, we found high levels of $D R B$ diversity in Qinling $R$. roxellana populations that suffered a rapid reduction in numbers and severe population fragmentation over the past several decades. We found several lines of evidence that suggest balancing selection has been acting on $D R B$ variation in this species.

First, in all three populations, population genetics analysis showed that neutral variation is relatively lower than variation for adaptive $M H C$ genes. The different diversity patterns of $M H C$ and neutral genes suggest that selection rather than neutral demographic processes (such as genetic drift, bottleneck and/or founder effect) results in a high level of genetic diversity for adaptive $M H C$ genes $[1,9,17]$. According to Huang et al. [28], although the Qinling $R$. roxellana populations suffered a rapid reduction in population size [23], there is no evidence of a past genetic bottleneck. Pan et al. [27] found 


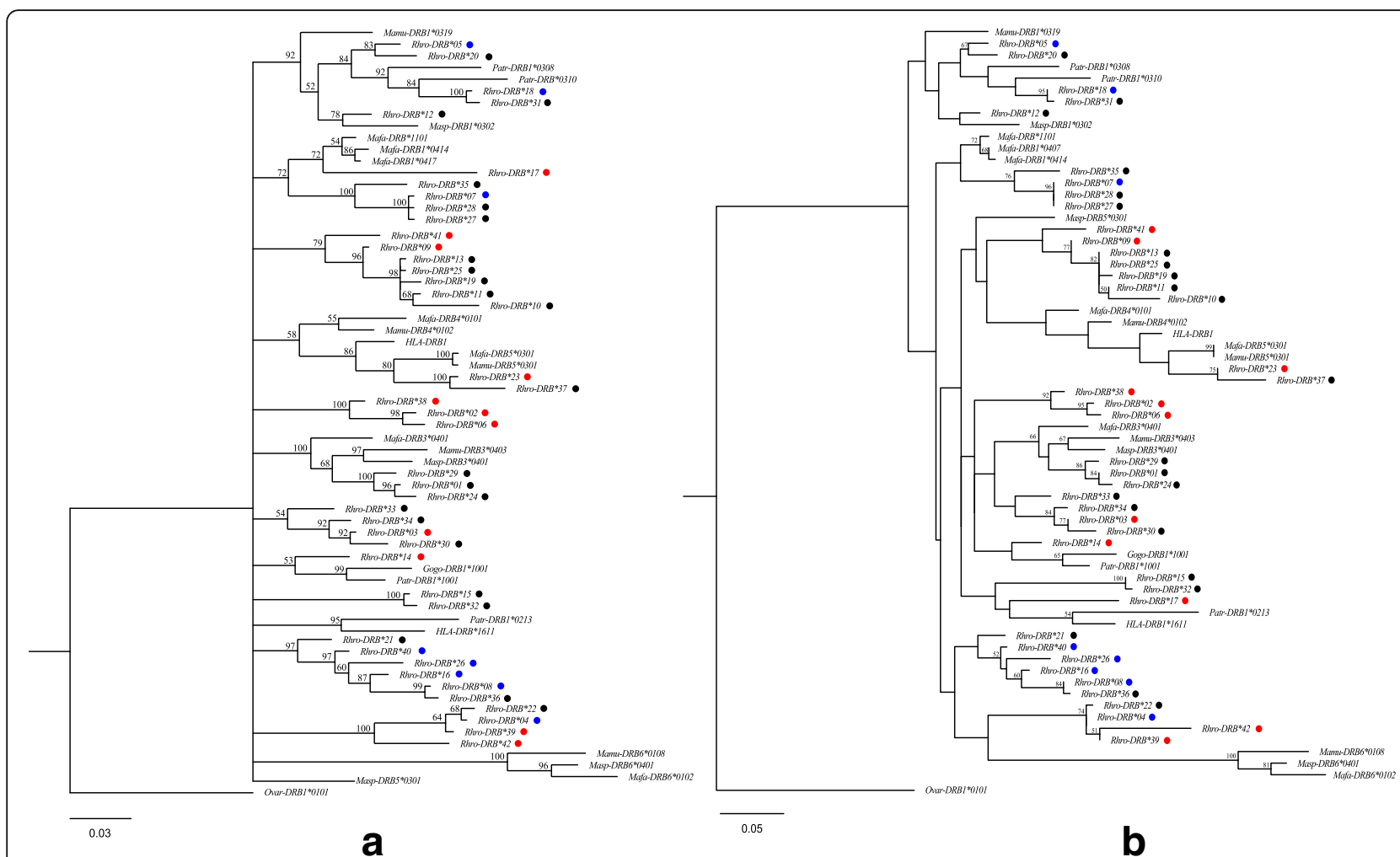

Fig. 2 Phylogenetic relationships of Rhro-DRB alleles conducted using Bayesian approach (a) and maximum likelihood method (b). Orthologous sequences from Ovis aries (Ovar-DRB1*0101), Macaca fascicularis (Mafa-DRB), Macaca mulatta (Mamu-DRB), Mandrillus sphinx (Masp-DRB), Pan troglodytes (Patr- $\overline{D R B}$ ), Gorilla gorilla (Gogo-DRB) and Homo sapiens (HLA-DRB) were include in the analysis. Values on the branch are represented for the posterior probability of the Bayesian tree and the support rate of the ML tree. Sequences labeled with solid circles are DRB alleles from $R$. roxellana. Colored circles indicate sequences detected in the three study populations. Red ones indication Rhro-DRB1 alleles, while blue ones indicate Rhro-DRB2 alleles

much polymorphism in $R$. roxellana at the mitochondrial control region (D-loop), suggesting that any influence of a founder effect on genetic diversity is weak. Despite the strength of influence of genetic drift, past bottlenecks and/or founder effects in our study populations, these factors will reduce genetic diversity. Similar patterns of high variability of $M H C$ genes coupled with relatively low microsatellite variability occurs in natural populations of other vertebrate species. For example, the house finch (Carpodacus mexicanus), a species that has experienced past population decline due to a disease epidemic, has high multi-locus $M H C$ diversity but relatively moderate levels of variability at microsatellites [72]. A more extreme example is the San Nicholas Island fox (Urocyon littoralis dickeyi), a critically endangered species that has high MHC heterozygosity but has little if any microsatellite genetic variation [73].

Second, we found that the $M H C$ allele frequencies of the three populations were less differentiated than the microsatellite allele frequencies (Table 5). This is as predicted for a gene under balancing selection or one closely linked to such a locus [73]. In a relatively small geographic region, where populations experience similar pathogen mediated selective regimes, i.e. homogenous selection pressure, balancing selection tends to decrease the levels of among-population variation [10, 74]. Therefore, the low differentiation among $R$. roxellana populations in the Qinling Mountains might be a result of homogenous balancing selection.

Third, positive Tajima's $D$ values at two $D R B$ loci (Table 4) indicated that the number of alleles at intermediate frequency was higher than expected, possibly as a result of balancing selection and/or rapid population contact [40]. However, the Tajima's $D$ statistics do not differ significantly so we cannot reject the null hypothesis that the $D R B$ gene is under neutral selection. Even so, there is additional evidence supporting positive selection. Nucleotide sites under positive selection are expected to accumulate more non-synonymous than synonymous substitutions, eventually bringing about amino acid changes and corresponding functional changes in MHC proteins [60]. Such adaptive evolutionary processes, possibly due to pathogen-mediated balancing selection, should be evident at the ABS [60]. 
According to the proposed criteria, $R$. roxellana $D R B$ genes showed evidence of positive selection for diversification. Positive selection acted only on ABS codons, with a significantly increased level of non-synonymous substitutions, whereas non-ABS codons exhibited significant negative/purifying selection. Rates of nonsynonymous substitutions were 7.32 times higher in the $\mathrm{ABS}$ than the non-ABS. This suggests that the polymorphism due to positive selection, in functionally important regions of the MHC molecule, allows $R$. roxellana to respond to a wider range of pathogens.

Additionally, our six random sites model analysis in PAML V4.7 [46] revealed the existence of positive selection in the maximum likelihood method. Our results suggested that the models including selection (M2a, M3 and M8) matched $D R B$ alleles better than those without selection (Tables 7 and 8). Under the M2a and M8 models, the same two ABS sites (11F and $13 \mathrm{~S}$ ) were exposed to significant selection, whilst under the M3 model, 32 sites including most ABS sites (17 of 18) were detected as being under positive selection. Our results are in accordance with substitutions often occurring within a functionally important domain [60].

Further evidence for balancing selection was provided by our result showing trans-species evolution of the $D R B$ alleles. Pathogen mediated balancing selection can result in allele retention among species for long evolutionary time periods, resulting in similar or even identical alleles being shared among extant species [18]. Such balancing selection gives rise to patterns of gene trees for the $M H C$ that differ from species relationships, termed 'trans-species evolution' [75]. Evidence for trans-species evolution for the $M H C$ gene complex has been previously found in a variety of vertebrate taxa (fishes: [76]; amphibians: [60]; reptiles: [77]; birds: [78]; mammals: [48]). In this study, we present clear phylogenetic evidence of trans-species evolution of DRB sequences across $R$. roxellana, Macaca fascicularis, Macaca mulatta, Mandrillus sphinx, Pan troglodytes and even Homo sapiens (Fig. 2; Additional file 2: Figure S2). This suggests that due to balancing selection, some allelic lineages have been retained over long evolutionary time periods and certain alleles that are shared among species are older than the diversification time of species or even families.

\section{Conclusions}

Many wild species are threatened by a dramatic reduction in and fragmentation of habitat, geographic isolation, small and declining populations and a decrease in genetic diversity [24, 79-82]. Understanding the patterns of adaptive diversity of threatened species is crucial. $M H C$ genes play an important role in adaptive immunology, and are ideal markers to study adaptive evolution [18]. Many wild populations have suffered from a reduction in $M H C$ diversity after population decline [70]. However, in this study, we found high levels of $D R B$ diversity in three $R$. roxellana populations that have suffered a rapid reduction in numbers and severe population fragmentation over the past several decades. We also found evidence of pathogen-mediated balancing selection, which is likely to have contributed to maintaining $M H C$ polymorphism over time. Our study adds to information on $M H C$ genes and may assist in developing effective management strategies for $R$. roxellana. Our new data furthers our understanding of the role pathogen-mediated balancing selection has in maintaining variation in $M H C$ genes in small and fragmented populations of free-ranging vertebrates.

\section{Additional files}

\begin{abstract}
Additional file 1: Figure S1. Sequence alignments of the deduced amino acid sequences for exon 2 of Rhro-DRB sequences. DRB sequences were taken from this study and previously published research (Luo and Pan 2013) and were included in sequence alignments. Dots indicate identity with the first sequence." +" and "**on the alignment represents putative ABS and sites contact to TCR, respectively. The putative ABS and sites contact to TCR were both derived according to the structure of human DRB genes (Reche and Reinherz, 2003). (PDF 308 kb)

Additional file 2: Figure S2. Phylogenetic tree of Rhro-DRB alleles using the maximum parsimony method. Orthologous sequences from Ovis aries (Ovar-DRB1*0101), Macaca fascicularis (Mafa-DRB), Macaca mulatta (Mamu-DRB), Mandrillus sphinx (Masp-DRB), Pan troglodytes (Patr-DRB), Gorilla gorilla (Gogo-DRB) and Homo sapiens $(H L \bar{A}-D R B)$ were include in the analysis. Values on the branch are represented for the support rate of the MP tree. Sequences labeled with solid circles are DRB alleles from $R$. roxellana. Colored circles indicate sequences detected in the three study populations. Red ones indication Rhro-DRB1 alleles, while blue ones indicate Rhro-DRB2 alleles. (PDF 183 kb)
\end{abstract}

\section{Abbreviations}

MHC: major histocompatibility complexABSAntigen binding sitesTCRT-cell receptorFPFopingNSNingshanZZZhouzhiMLMaximum likelihoodMPMaximum parsimony

\section{Acknowledgements}

We thank the staff of the Shaanxi Key Laboratory for Animal Conservation for the samples provided and laboratory assistance. This study was supported by the National Natural Science Foundation of China (NSFC: 31730104 , 31770425, 31770411 and 31501872), the National Key Programme of Research and Development, the Ministry of Science and Technology of China (2016YFC0503202), and the Undergraduate Training Program for Innovation and Entrepreneurship (2018302). We thank the handling editor and the three reviewers for their constructive comments on earlier versions of this paper.

\section{Funding}

This project was funded by the National Natural Science Foundation of China (31730104, 31770425, 31770411 and 31501872), and the National Key Programme of Research and Development, the Ministry of Science and Technology of China (2016YFC0503202).

\section{Availability of data and materials}

All new sequences were submitted to GenBank (accession numbers: MF434639-MF434643). The dataset used and analyzed during the current study are available from the corresponding author on reasonable request.

\section{Authors' contributions}

$\mathrm{PZ}$ performed the statistical analysis, and drafted the manuscript. $\mathrm{PZ}, \mathrm{KH}, \mathrm{BYZ}$ and DC carried out the molecular genetic studies. KH, FL, XGQ and DWD 
edited the manuscript. STG participated in the sampling. BGL conceived the study, participated in its design and helped to draft the manuscript. All authors read and approved the final manuscript.

\section{Ethics approval and consent to participate}

All samples were collected with an ethical permission from the animal care committee of the Wildlife Protection Society of China (SL-2012-42).

\section{Consent for publication}

Not applicable.

\section{Competing interests}

The authors declare that they have no competing interests.

\section{Publisher's Note}

Springer Nature remains neutral with regard to jurisdictional claims in published maps and institutional affiliations.

\section{Author details}

'Shaanxi Key Laboratory for Animal Conservation, College of Life Sciences, Northwest University, Xi'an, China. ${ }^{2}$ Middle School Affiliated to Northwest University, Xi'an, China. ${ }^{3}$ Xi'an Branch of Chinese Academy of Science, Xi'an, China.

\section{Received: 20 July 2017 Accepted: 6 March 2018}

\section{Published online: 13 March 2018}

\section{References}

1. Eizaguirre C, Lenz TL, Kalbe M, Rapid MM. Adaptive evolution of MHC genes under parasite selection in experimental vertebrate populations. Nat Commun. 2012;3:621.

2. Ryan $\mathrm{SO}, \mathrm{Cobb} \mathrm{BA}$. Roles for major histocompatibility complex glycosylation in immune function. Semin Immunopathol. 2012;34:425-41.

3. Klein J. Natural history of the major histocompatibility complex. New York: John Wiley and Sons; 1986

4. Wolfert MA, Boons G-J. Adaptive immune activation: glycosylation does matter. Nat Chem Biol. 2013;9:776-84.

5. Neefjes J, Jongsma MLM, Paul P, Bakke O. Towards a systems understanding of MHC class I and MHC class II antigen presentation. Nat Rev Immunol. 2011;11:823-36.

6. Castro-Prieto A, Wachter B, Sommer S. Cheetah paradigm revisited: MHC diversity in the world's largest free-ranging population. Mol Biol Evol. 2011;28:1455-68.

7. Goda N, Mano T, Kosintsev P, Vorobiev A, Masuda R. Allelic diversity of the MHC class II DRB genes in brown bears (Ursus arctos) and a comparison of DRB sequences within the family Ursidae. Tissue Antigens. 2010;76:404-10.

8. Huchard E, Knapp LA, Wang JL, Raymond M, Cowlishaw G. MHC, mate choice and heterozygote advantage in a wild social primate. Mol Ecol. 2010;19:2545-61.

9. Spurgin LG, Richardson DS. How pathogens drive genetic diversity: MHC, mechanisms and misunderstandings. Proc R Soc Lond B Biol Sci. 2010;277:979-88.

10. Ashby B, Boots M. Multi-mode fluctuating selection in host-parasite coevolution. Ecol Lett. 2017;20:357-65.

11. Hedrick PW. What is the evidence for heterozygote advantage selection? Trends Ecol Evol. 2012;27:698-704.

12. Yang ZH, Bielawski JP. Statistical methods for detecting molecular adaptation. Trends Ecol Evol. 2000;15:496-503.

13. Kimura M. The neutral theory of molecular evolution. Cambridge: Cambridge University Press; 1983.

14. McCann HC, Nahal H, Thakur S, Guttman DS. Identification of innate immunity elicitors using molecular signatures of natural selection. Proc Natl Acad Sci U S A. 2012;109:4215-20.

15. Kosakovsky Pond SL, Frost SDW. Not so different after all: a comparison of methods for detecting amino acid sites under selection. Mol Biol Evol. 2005; 22:1208-22.

16. Klein J, Sato A, Nikolaidis N. MHC, TSP, and the origin of species: from immunogenetics to evolutionary genetics. Annu Rev Genet. 2007;41:281-304.

17. Charlesworth D. Balancing selection and its effects on sequences in nearby genome regions. PLoS Genet. 2006;2:e64.

18. Bernatchez $L$, Landry C. MHC studies in nonmodel vertebrates: what have we learned about natural selection in 15 years? J Evol Biol. 2003;16:363-77.
19. Sutton JT, Nakagawa S, Robertson BC, Jamieson IG. Disentangling the roles of natural selection and genetic drift in shaping variation at $\mathrm{MHC}$ immunity genes. Mol Ecol. 2011;20:4408-20.

20. Oosterhout CV, Joyce DA, Cummings SM, Blais J, Barson NJ, Ramnarine IW, et al. Balancing selection, random genetic drift, and genetic variation at the major histocompatibility complex in two wild populations of guppies (Poecilia reticulata). Evolution. 2006:60, 2562.

21. Li BG, Pan RL, Oxnard CE. Extinction of snub-nosed monkeys in China during the past 400 years. Int J Primatol. 2002;23:1227-44.

22. Qi XG, Garber PA, Ji WH, Huang ZP, Huang K, Zhang P, et al. Satellite telemetry and social modeling offer new insights into the origin of primate multilevel societies. Nat Commun. 2014;5:5296.

23. Long YC, Richardson M. Rhinopithecus roxellana. The IUCN Red List of Threatened Species. 2008:http://www.iucnredlist.org. Accessed 10 June 2017.

24. Dixo M, Metzger JP, Morgante JS, Zamudio KR. Habitat fragmentation reduces genetic diversity and connectivity among toad populations in the Brazilian Atlantic coastal Forest. Biol Conserv. 2009;142:1560-9.

25. Sommer $\mathrm{S}$. The importance of immune gene variability (MHC) in evolutionary ecology and conservation. Front Zool. 2005;2:16.

26. Luo MF, Liu ZJ, Pan HJ, Zhao L, Li M. Historical geographic dispersal of the golden snub-nosed monkey (Rhinopithecus roxellana) and the influence of climatic oscillations. Am J Primatol. 2012;74:91-101.

27. Pan D, Hu HX, Meng SJ, Men ZM, Fu YX, Zhang YP. High polymorphism level in Rhinopithecus roxellana. Int J Primatol. 2009;30:337-51.

28. Huang K, Guo ST, Cushman SA, Dunn DW, Qi XG, Hou R, et al. Population structure of the golden snub-nosed monkey Rhinopithecus roxellana in the Qinling Mountains, Central China. Integr Zool. 2016;11:350-60.

29. Walsh PS, Metzger DA, Higuchi R. Chelex 100 as a medium for simple extraction of DNA for PCR-based typing from forensic material. BioTechniques. 1991;10:506-13.

30. Luo MF, Pan HJ. MHC II DRB variation and trans-species polymorphism in the golden snub-nosed monkey (Rhinopithecus roxellana). Chin Sci Bull. 2013:58:2119-27.

31. Larkin MA, Blackshields G, Brown NP, Chenna RM, Mcgettigan PA, Mcwilliam $H$, et al. Clustal W. Clustal X version 2.0. Bioinformatics. 2007;23:2947-8.

32. Zhou XM, Wang BS, Pan Q, Zhang JB, Kumar S, Sun XQ, et al. Wholegenome sequencing of the snub-nosed monkey provides insights into folivory and evolutionary history. Nat Genet. 2014;46:1303-10.

33. Kirkpatrick S, Gekatt CD, Vecchi MP. Optimization by simulated annealing. Science. 1983;220:671-80.

34. Kumar S, Stecher G, Tamura K. MEGA7: molecular evolutionary genetics analysis version 7.0 for bigger datasets. Mol Biol Evol. 2016;33:1870-4.

35. Rousset F. GENEPOP'007: a complete re-implementation of the genepop software for windows and Linux. Mol Ecol Resour. 2008;8:103-6.

36. Goudet J. FSTAT, a program to estimate and test gene diversities and fixation indices (version 2.9.3). 2001. https://www2.unil.ch/popgen/ softwares/fstat.htm.

37. Kalinowski ST, Taper ML, Marshall TC. Revising how the computer program CERVUS accommodates genotyping error increases success in paternity assignment. Mol Ecol. 2007:16:1099-106.

38. Peakall R, Smouse PE. GENALEX 6: genetic analysis in excel. Population genetic software for teaching and research. Mol Ecol Notes. 2006;6:288-95.

39. Librado P, Rozas J. DnaSP v5: a software for comprehensive analysis of DNA polymorphism data. Bioinformatics. 2009;25:1451-2.

40. Bamshad MJ, Mummidi S, Gonzalez E, Ahuja SS, Dunn DM, Watkins WS, et al. A strong signature of balancing selection in the $5^{\prime}$ cis-regulatory region of CCR5. Proc Natl Acad Sci U S A. 2002;99:10539-44.

41. Darriba D, Taboada GL, Doallo R, Posada D. jModelTest 2: more models, new heuristics and parallel computing. Nat Methods. 2012;9:772.

42. Ronquist F, Huelsenbeck JP. MrBayes 3: Bayesian phylogenetic inference under mixed models. Bioinformatics. 2003;19:1572-4.

43. Guindon S, Dufayard J-F, Lefort V, Anisimova M, Hordijk W, Gascuel O. New algorithms and methods to estimate maximum-likelihood phylogenies: assessing the performance of PhyML 3.0. Syst Biol. 2010;59:307-21.

44. Nei M, Gojobori T. Simple methods for estimating the numbers of synonymous and nonsynonymous nucleotide substitutions. Mol Biol Evol. 1986;3:418-26.

45. Reche PA, Reinherz EL. Sequence variability analysis of human class I and class II MHC molecules: functional and structural correlates of amino acid polymorphisms. J Mol Biol. 2003;331:623-41.

46. Yang ZH. PAML 4: phylogenetic analysis by maximum likelihood. Mol Biol Evol. 2007;24:1586-91. 
47. Klein J, Bontrop RE, Dawkins RL, Erlich HA, Gyllensten UB, Heise ER, et al. Nomenclature for the major histocompatibility complexes of different species: a proposal. Immunogenetics. 1990;31:217-9.

48. Axtner J, Sommer S. Gene duplication, allelic diversity, selection processes and adaptive value of MHC class II DRB genes of the bank vole, Clethrionomys glareolus. Immunogenetics. 2007:59:417-26.

49. Go Y, Satta Y, Kawamoto Y, Rakotoarisoa G, Randrianjafy A, Koyama N, et al. Frequent segmental sequence exchanges and rapid gene duplication characterize the MHC class I genes in lemurs. Immunogenetics. 2003;55: 450-61.

50. Yang G, Yan J, Zhou K, Wei F. Sequence variation and gene duplication at MHC DQB loci of Baiji (Lipotes vexillifer), a Chinese river dolphin. J Hered. 2005:96:310-7.

51. Piontkivska $H$, Nei M. Birth-and-death evolution in primate MHC class I genes: divergence time estimates. Mol Biol Evol. 2003;20:601-9.

52. Hughes AL, Yeager M. Natural selection at major histocompatibility complex loci of vertebrates. Annu Rev Genet. 1998:32:415-35.

53. Doxiadis GGM, Otting N, de Groot NG, Noort R, Bontrop RE. Unprecedented polymorphism of $M h c-D R B$ region configurations in rhesus macaques. J Immunol. 2000;164:3193-9.

54. Doxiadis GGM, Rouweler AJM, de Groot NG, Louwerse A, Otting N, Verschoor EJ, et al. Extensive sharing of MHC class II alleles between rhesus and cynomolgus macaques. Immunogenetics. 2006;58:259.

55. Huchard E, Cowlishaw G, Raymond M, Weill M, Knapp LA. Molecular study of Mhc-DRB in wild chacma baboons reveals high variability and evidence for trans-species inheritance. Immunogenetics. 2006;58:805-16.

56. Schad J, Sommer S, Ganzhorn JU. MHC variability of a small lemur in the littoral forest fragments of southeastern Madagascar. Conserv Genet. 2004;5: 299-309.

57. Schwensow N, Fietz J, Dausmann KH, Sommer S. Neutral versus adaptive genetic variation in parasite resistance: importance of major histocompatibility complex supertypes in a free-ranging primate. Heredity. 2007;99:265-77.

58. Fukami-Kobayashi K, Shiina T, Anzai T, Sano K, Yamazaki M, Inoko H, et al. Genomic evolution of MHC class I region in Primates. Proc Natl Acad Sci U S A. 2005;102:9230-4.

59. Cohen S. Strong positive selection and habitat-specific amino acid substitution patterns in $\mathrm{MHC}$ from an estuarine fish under intense pollution stress. Mol Biol Evol. 2002;19:1870-80.

60. Kiemnec-Tyburczy KM, Richmond JQ, Savage AE, Lips KR, Zamudio KR. Genetic diversity of MHC class I loci in six non-model frogs is shaped by positive selection and gene duplication. Heredity. 2012;109:146-55.

61. Alcaide M, Edwards SV, Negro JJ, Serrano D, Tella JL. Extensive polymorphism and geographical variation at a positively selected MHC class II B gene of the lesser kestrel (Falco naumanni). Mol Ecol. 2008;17:2652-65.

62. Wan QH, Zhu L, Wu H, Fang SG. Major histocompatibility complex class ॥ variation in the giant panda (Ailuropoda melanoleuca). Mol Ecol. 2006;15:2441-50.

63. Zhang P, Song XY, Dunn DW, Huang K, Pan RL, Chen D, et al. Diversity at two genetic loci associated with the major histocompatibility complex in the golden snub-nosed monkey (Rhinopithecus roxellana). Biochem Syst Ecol. 2016;68:243-9.

64. Wang JL. Unbiased relatedness estimation in structured populations Genetics. 2011;187:887-901.

65. Ritland K. Inferences about inbreeding depression based on changes of the inbreeding coefficient. Evolution. 1990:44:1230-41.

66. Birchler JA, Yao H, Chudalayandi S. Unraveling the genetic basis of hybrid vigor. Proc Natl Acad Sci U S A. 2006;103:12957-8.

67. Bearhop S, Fiedler W, Furness RW, Votier SC, Waldron S, Newton J, et al. Assortative mating as a mechanism for rapid evolution of a migratory divide. Science. 2005:310:502-4.

68. Wagner AP, Creel S, Kalinowski ST. Estimating relatedness and relationships using microsatellite loci with null alleles. Heredity. 2006:97:336-45.

69. Fumagalli M, Sironi M, Pozzoli U, Ferrer-Admettla A, Pattini L, Nielsen R. Signatures of environmental genetic adaptation pinpoint pathogens as the main selective pressure through human evolution. PLoS Genet. 2011;7:e1002355.

70. Eimes JA, Bollmer JL, Whittingham LA, Johnson JA, Van Oosterhout C, Dunn PO. Rapid loss of MHC class II variation in a bottlenecked population is explained by drift and loss of copy number variation. J Evol Biol. 2011;24:1847-56.

71. Miller HC, Lambert DM. Genetic drift outweighs balancing selection in shaping post-bottleneck major histocompatibility complex variation in New Zealand robins (Petroicidae). Mol Ecol. 2004;13:3709-21.
72. Hawley DM, Fleischer RC. Contrasting epidemic histories reveal pathogenmediated balancing selection on class $\| \mathrm{MHC}$ diversity in a wild songbird. PLoS One. 2012;7:e30222.

73. Aguilar A, Roemer G, Debenham S, Binns M, Garcelon D, Wayne RK, High $\mathrm{MHC}$. Diversity maintained by balancing selection in an otherwise genetically monomorphic mammal. Proc Natl Acad Sci U S A. 2004;101: 3490-4.

74. Hall AR, Scanlan PD, Morgan AD, Buckling A. Host-parasite coevolutionary arms races give way to fluctuating selection. Ecol Lett. 2011;14:635-42.

75. Garrigan D, Hedrick PW. Perspective: detecting adaptive molecular polymorphism: lessons from the MHC. Evolution. 2003;57:1707-22.

76. Ottová E, Šimková A, Martin J-F, De Bellocq JG, Gelnar M, Allienne J-F, et al. Evolution and trans-species polymorphism of MHC class $\| \beta$ genes in cyprinid fish. Fish Shellfish Immunol. 2005;18:199-222.

77. Stiebens VA, Merino SE, Chain FJ, Eizaguirre C. Evolution of MHC class I genes in the endangered loggerhead sea turtle (Caretta caretta) revealed by 454 amplicon sequencing. BMC Evol Biol. 2013;13:95.

78. Richardson DS, Westerdahl H. MHC diversity in two Acrocephalus species: the outbred great reed warbler and the inbred Seychelles warbler. Mol Ecol. 2003;12:3523-9.

79. Lindsay DL, Barr KR, Lance RF, Tweddale SA, Hayden TJ, Leberg PL. Habitat fragmentation and genetic diversity of an endangered, migratory songbird, the golden-cheeked warbler (Dendroica chrysoparia). Mol Ecol. 2008;17: 2122-33.

80. Keyghobadi N, Roland J, Matter SF, Strobeck C. Among- and within-patch components of genetic diversity respond at different rates to habitat fragmentation: an empirical demonstration. Proc R Soc Lond B Biol Sci. 2005;272:553-60.

81. Segelbacher G, Manel S, Tomiuk J. Temporal and spatial analyses disclose consequences of habitat fragmentation on the genetic diversity in capercaillie (Tetrao urogallus). Mol Ecol. 2008;17:2356-67.

82. Rosas F, Quesada M, Lobo JA, Sork VL. Effects of habitat fragmentation on pollen flow and genetic diversity of the endangered tropical tree Swietenia humilis (Meliaceae). Biol Conserv. 2011;144:3082-8.

\section{Submit your next manuscript to BioMed Central and we will help you at every step:}

- We accept pre-submission inquiries

- Our selector tool helps you to find the most relevant journal

- We provide round the clock customer support

- Convenient online submission

- Thorough peer review

- Inclusion in PubMed and all major indexing services

- Maximum visibility for your research

Submit your manuscript at www.biomedcentral.com/submit 